\title{
Stellar and dust properties of local elliptical galaxies: clues to the onset of nuclear activity (Research Note)
}

\author{
Y. Zhang ${ }^{1}$, Q.-S. Gu ${ }^{1}$, and L. C. $\mathrm{Ho}^{2}$ \\ 1 Department of Astronomy, Nanjing University, Nanjing 210093, PR China \\ e-mail: zhangyu19842007@hotmail.com; qsgu@nju.edu.cn \\ 2 The Observatories of the Carnegie Institution of Washington, 813 Santa Barbara Street, Pasadena, CA 91101, USA \\ e-mail: 1ho@ociw.edu
}

Received 27 February 2008 / Accepted 4 June 2008

\section{ABSTRACT}

\begin{abstract}
Aims. We study the stellar and dust properties of a well-defined sample of local elliptical galaxies to investigate the relationship between host galaxy properties and nuclear activity.

Methods. We have selected a complete sample of 45 ellipticals from the Palomar spectroscopic survey of nearby galaxies, which includes 20 low-luminosity active galactic nuclei classified as LINERs and 25 inactive galaxies. Using a stellar population synthesis method, we compared the derived stellar population properties of the LINER to the inactive subsamples. We also studied the dust and stellar surface brightness distributions of the central regions of these galaxies using high-resolution images obtained with the Hubble Space Telescope.

Results. Compared with the inactive subsample, ellipticals hosting LINERs share similar total optical and near-infrared luminosity, central stellar velocity dispersions, and nuclear stellar populations as judged from their luminosity-weighted ages and metallicities. LINERs, on the other hand, have a larger fraction of core-type central surface brightness profiles and a much higher frequency of circumnuclear dust structures.

Conclusions. Our results support the suggestion that LINERs are powered by low-luminosity AGNs rather than by young or intermediate-age stars. Nuclear activity in nearby elliptical galaxies seems to occur primarily in those systems where enough cold interstellar material has managed to accumulate, perhaps via cooling condensations from hot gas.
\end{abstract}

Key words. galaxies: elliptical and lenticular, $\mathrm{cD}$ - galaxies: active - galaxies: stellar content

\section{Introduction}

Supermassive black holes (SMBHs) are ubiquitous in elliptical galaxies. The discovery of a tight correlation between black hole mass and the stellar velocity dispersion of the bulge of the host galaxy (Gebhardt et al. 2000; Ferrarese \& Merritt 2000) suggests that the formation and growth of central black holes are an integral part of the formation of galactic bulges. However, the majority of SMBHs in nearby ellipticals are not very active. In the Palomar spectroscopic survey of nearby galaxies (Ho et al. 1995, 1997a), only about $50 \%$ of the ellipticals show detectable emission-line nuclei, most of which $(\sim 87 \%)$ are classified as low-ionization nuclear emission-line regions (LINERs; Heckman 1980; see Ho 2008, for a review).

The physical origin of LINERs has been intensively debated. A variety of recent observations, however, have provided convincing evidence that LINERs are low-luminosity active galactic nuclei (AGNs). This includes the detection of broad $\mathrm{H} \alpha$ emission (Ho et al. 1997b), some of which show double-peaked profiles (Ho et al. 2000; Shields et al. 2000; Barth et al. 2001) or are polarized (Barth et al. 1999a,b), ultraviolet variability (Maoz et al. 2005), compact X-ray cores (e.g., Ho et al. 2001; Terashima \& Wilson 2003), and compact radio cores (e.g., Nagar et al. 2005). Ho (2008) suggests that nearly all LINERs are genuine AGNs with low accretion rates.

The low accretion rates of the SMBHs in nearby ellipticals probably stem from the lack of enough cold gas to fuel the central engine, as it is generally accepted that nearby elliptical galaxies contain mostly old stars and not much cold interstellar material. However, apart from the high detection rate of nuclear optical emission lines in spectroscopic surveys (Phillips et al. 1986; Ho et al. 1997a), H $\alpha+$ [N II] narrowband imaging has shown that elliptical galaxies certainly have plenty of extended warm $\left(\sim 10^{4} \mathrm{~K}\right)$ gas (e.g., Shields 1991). By coadding images from the Infrared Astronomy Satellite survey scans, Knapp et al. (1989) show that $250 \%$ of ellipticals contain cool dust. Temi et al. (2004) find that in 16 elliptical galaxies observed with the Infrared Space Observatory the dust masses are on average 10 times larger than those previously estimated from Infrared Astronomy Satellite observations. Most recently, Kaneda et al. (2005) have even detected polycyclic aromatic hydrocarbon emission features in four elliptical galaxies with the Spitzer Infrared Spectrograph. All these results suggest that the presence of a significant amount of interstellar material in elliptical galaxies is quite common, although its origin is still uncertain.

We attempt to determine the factor(s) that control the onset of nuclear activity by comparing the stellar population, central light distribution, and dust content for ellipticals with different levels of nuclear activity drawn from the Palomar spectroscopic survey of nearby galaxies (Ho et al. 1995, 1997a). This paper is organized as follows. The sample and analysis method are described in Sect. 2; the results of the stellar population synthesis 
are presented in Sect. 3; the discussion and conclusions are given in Sect. 4.

\section{Sample and method of analysis}

The sample for this study was selected from the Palomar optical spectroscopic survey (Ho et al. 1995, 1997a), which comprises all nearby galaxies brighter than $B_{\mathrm{T}}=12.5 \mathrm{mag}$ in the northern hemisphere. It presents a fair representation of the local galaxy population. This is an ideal sample because it is statistically complete and contains both active ellipticals and inactive objects, drawn self-consistently from the same parent sample, that can serve as a control sample. The optical spectra are described in detail in Ho et al. (1995). Two spectra are available for each galaxy: the blue section covers $\sim 4230-5110 \AA$ with a $F W H M$ spectral resolution of $4 \AA$, and the red section covers $\sim 6210-6860 \AA$ with a $F W H M$ resolution of $2.5 \AA$. We select all ellipticals from the parent sample classified as either LINERs or absorption-line (inactive) sources, according to the criteria given in Ho et al. (1997a). We reject NGC 6702 because it only has the red spectrum, and two spheroidals (NGC 147 and NGC 205) because they have a different formation history than normal ellipticals (Kormendy 1985). We include NGC 221 (M32), even though its absolute $B$-band magnitude is less than $-18 \mathrm{mag}$, because it shares properties similar to those of normal ellipticals (Kormendy 1985; Ferguson \& Binggeli 1994). Since the vast majority of the active sources are LINERs, we further removed 4 Seyferts and 5 transition objects. The final sample, whose global properties are summarized in Table 1, comprises 20 LINERs and 25 inactive ellipticals.

We modeled the stellar population of our sample using a modified version of the spectral population synthesis code, starlight $^{1}$, (Cid Fernandes et al. 2004; Gu et al. 2006). The code does a search for the best-fitting linear combination of 45 simple stellar populations (SSPs) -15 ages and 3 metallicities $\left(0.2 Z_{\odot}\right.$, $\left.1 Z_{\odot}, 2.5 Z_{\odot}\right)$ - provided by Bruzual \& Charlot (2003) to match a given observed spectrum $O_{\lambda}$. The model SSP spectra cover $3200 \AA$ to $9500 \AA$ with a $F W H M$ spectral resolution of $\sim 3 \AA$ (Bruzual \& Charlot 2003, BC03 hereafter). The synthesized spectrum is given by (Cid Fernandes et al. 2004):

$M_{\lambda}=M_{\lambda_{0}}\left(\sum_{i=1}^{N_{*}} x_{i} b_{i, \lambda} r_{\lambda}\right) \otimes G\left(v_{*}, \sigma_{*}\right)$,

where $M_{\lambda}$ is the synthesized spectrum, $M_{\lambda_{0}}$ is the synthesized flux at normalization wavelength $\lambda_{0}$ (4750 $\AA$ in this work), $x_{i}$ is the contribution of the $i$ th SSP, $b_{i, \lambda}$ is the spectrum of the $i$ th SSP normalized at $\lambda_{0}$, and $r_{\lambda}=10^{-0.4\left(A_{\lambda}-A_{\lambda_{0}}\right)}$ is the reddening term. The Cardelli et al. (1989) extinction law with $R_{\mathrm{V}}=3.1$ is adopted. $G\left(v_{*}, \sigma_{*}\right)$ is the line-of-sight stellar velocity distribution, modeled as a Gaussian distribution centered at $v_{*}$ and broadened by dispersion $\sigma_{*}$. The match between the model and the observed spectrum is calculated by $\chi^{2}\left(x, M_{\lambda_{0}}, A_{\mathrm{V}}, v_{*}, \sigma_{*}\right)=$ $\sum_{i=1}^{N_{*}}\left[\left(O_{\lambda}-M_{\lambda}\right) w_{\lambda}\right]^{2}$, where the weight spectrum, $w_{\lambda}$, is defined as the inverse of the noise of $O_{\lambda}$. For more details, see Cid Fernandes et al. (2004, 2005).

In order to account for the possibility of a weak AGN continuum (see Sect. 5.1 of Ho 2008), we add an additional power-law (PL) component $\left(f_{v} \propto v^{-1.5}\right)$ during the fitting. Our fitting results show that, with the exception of NGC 315 and NGC 3193, the contribution from this PL component is negligible (less

${ }^{1}$ http://www.starlight.ufsc.br/ than $2 \%$ ). Strong emission lines, such as $\mathrm{H} \beta$, [O III] $\lambda \lambda 4959$, $5007, \mathrm{H} \alpha$, and [N II] $\lambda \lambda 6548,6583$, are masked during the fitting process. The fitting procedure automatically determines a bestfitting stellar velocity dispersion $\left(\sigma_{*}\right)$, taking into account the instrumental resolutions of both the Palomar and model library spectra. Cid Fernandes et al. (2004) estimate that the $\sigma_{*}$ values derived from the code has a typical uncertainty of $\sim 20 \mathrm{~km} \mathrm{~s}^{-1}$.

Figures 1 and 2 show two fitting examples, for the LINER NGC 2768 and the inactive elliptical NGC 4648, respectively. The emission-line fluxes and line-intensity ratios measured in the residual spectra for the entire sample show excellent agreement (differences $<10 \%$ ) with those given in the original Palomar survey.

\section{Results}

\subsection{Distance, luminosity, and stellar velocity dispersion}

The distance and absolute blue magnitude of each galaxy, taken from Ho et al. (1997a), are listed in Table 1. In Fig. 3a we show the cumulative distribution of distance for the two subsamples. We find that LINERs and inactive ellipticals have similar distance distributions, with average distances of 29.9 and 25.7 Mpc, respectively. The LINER subsample contains the most distant object in the study, NGC 2832 at $D=91.6 \mathrm{Mpc}$; if we exclude this object, the average distance for the LINERs becomes 27.6 Mpc. We use the two-sample Kolmogorov-Smirnov (KS) statistical task kolmov in IRAF $^{2}$ to check whether the two subsamples are drawn from the same parent distribution. The probability of rejecting the null hypothesis that the two distributions are the same is $P_{\text {null }}=94 \%$. Thus, distance effects between the two subsamples will not introduce any significant biases into our results.

The two subsamples also have very similar total luminosities, both in the optical and near-infrared bands. Figure $3 \mathrm{~b}$ shows the cumulative distributions of absolute blue magnitude. LINERs have $M_{\mathrm{B}}$ ranging from -18.96 to -22.24 mag, with an average value of $-20.69 \mathrm{mag}$, while inactive Es range from $M_{\mathrm{B}}=-15.51$ to $-21.64 \mathrm{mag}$, with an average value of -20.11 mag. A KS test yields $P_{\text {null }}=38 \%$. We also derive absolute magnitudes in the $K_{\mathrm{s}}$ band, $M_{K_{\mathrm{s}}}$, using data taken from the Two Micron All Sky Survey (Skrutskie et al. 2006). Figure 3c shows that the absolute $K_{\mathrm{s}}$-band magnitudes of LINERs are similar to those of inactive Es: LINERs have an average $M_{K_{\mathrm{s}}}=$ $-24.54 \mathrm{mag}$, to be compared with $M_{K_{\mathrm{s}}}=-23.90 \mathrm{mag}$ for inactive Es. The near-infrared luminosity of the active sources have a slight tendency to be larger than those of the inactive sources, but a KS test yields $P_{\text {null }} \approx 5 \%$, which is formally only barely statistically significant. Moreover, this conclusion is not very robust. If we exclude the two most luminous objects from the active sample (NGC 315 and NGC 2832) and the least luminous object from the inactive sample (NGC 221), the significance drops to $P_{\text {null }} \approx 14 \%$.

Not surprisingly, the similarity between the two subsamples extends to the stellar velocity dispersions derived from our synthesis fitting (data in Col. 6 of Table 1), whose cumulative distributions are shown in Fig. 3d. For LINERs, the velocity dispersion ranges from 144 to $331 \mathrm{~km} \mathrm{~s}^{-1}$, with an average value of $\left\langle\sigma_{*}\right\rangle=251 \mathrm{~km} \mathrm{~s}^{-1}$; the corresponding values for inactive Es are $90<\sigma_{*}<349 \mathrm{~km} \mathrm{~s}^{-1}$, with $\left\langle\sigma_{*}\right\rangle=211 \mathrm{~km} \mathrm{~s}^{-1}$. A KS test for

\footnotetext{
2 IRAF is distributed by the National Optical Astronomy Observatories, which are operated by the Association of Universities for Research in Astronomy, Inc., under cooperative agreement with the
} National Science Foundation. 
Table 1. Global and central properties.

\begin{tabular}{|c|c|c|c|c|c|c|c|c|c|c|}
\hline LINERs & $D(\mathrm{Mpc})$ & $M_{\mathrm{B}}(\mathrm{mag})$ & $M_{K_{\mathrm{S}}}(\mathrm{mag})$ & Class & $\sigma_{*}\left(\mathrm{~km} \mathrm{~s}^{-1}\right)$ & $A_{\mathrm{V}}(\mathrm{mag})$ & Profile & Ref. & Dust Properties & Ref. \\
\hline NGC 315 & 65.8 & -22.22 & -26.14 & L1.9 & 322 & 0.42 & & & Dust disk & 2 \\
\hline NGC 1052 & 17.8 & -19.90 & -23.80 & L1.9 & 240 & 0.04 & core & 1,9 & Has dust & 3 \\
\hline NGC 2768 & 23.7 & -21.17 & -24.88 & L2 & 170 & 0.32 & & & Spiral dust lanes & 4 \\
\hline NGC 2832 & 91.6 & -22.24 & -26.11 & L2:: & 329 & 0.02 & core & 1,10 & No dust & 5 \\
\hline NGC 3193 & 23.2 & -20.10 & -23.85 & L2: & 193 & -0.02 & core & 1,8 & No dust & 6 \\
\hline NGC 3226 & 23.4 & -19.40 & -23.28 & L1.9 & 227 & 0.61 & & & Dust disk & 2 \\
\hline NGC 3379 & 8.1 & -19.36 & -23.27 & L2/T2:: & 237 & -0.17 & core & 1,4 & Dust ring or disk & 4 \\
\hline NGC 3608 & 23.4 & -20.16 & -23.75 & $\mathrm{~L} 2 / \mathrm{S} 2:$ & 218 & -0.10 & core & 1,4 & Dust ring or disk & 4 \\
\hline NGC 4261 & 35.1 & -21.37 & -25.46 & L2 & 331 & 0.16 & core & 1,3 & Dust disk & 2 \\
\hline NGC 4278 & 9.7 & -18.96 & -22.75 & L1.9 & 269 & -0.11 & core & 1,4 & Spiral dust lanes & 4 \\
\hline NGC 4374 & 16.8 & -21.12 & -24.90 & L2 & 246 & 0.96 & core & 1,3 & Dust lanes & 3 \\
\hline NGC 4486 & 16.8 & -21.64 & -25.31 & L2 & 328 & 0.04 & core & 1,11 & No dust & 7 \\
\hline NGC 4494 & 9.7 & -19.38 & -22.94 & L2:: & 144 & 0.14 & PL & 1,8 & Dust ring or disk & 4 \\
\hline NGC 4589 & 30.0 & -20.71 & -24.63 & L2 & 243 & 0.34 & core & 1,4 & Chaotic dust patches & 4 \\
\hline NGC 4636 & 17.0 & -20.72 & -24.73 & L1.9 & 215 & 0.14 & core & 1,3 & Dust lanes & 7 \\
\hline NGC 5077 & 40.6 & -20.83 & -24.83 & L1.9 & 266 & 0.04 & core & 1,8 & Dust filaments & 6,8 \\
\hline NGC 5322 & 31.6 & -21.46 & -25.34 & L2:: & 232 & -0.08 & & & Dust ring or disk & 4 \\
\hline NGC 5813 & 28.5 & -20.85 & -24.86 & L2: & 257 & 0.21 & core & 1,4 & Dust disk & 6 \\
\hline NGC 5982 & 38.7 & -20.89 & -24.79 & L2:: & 267 & -0.12 & core & 1,4 & No dust & $2,3,6$ \\
\hline NGC 7626 & 45.6 & -21.23 & -25.26 & L2:: & 286 & 0.22 & int & 1,3 & Dust ring or disk & 2,4 \\
\hline average & 29.9 & -20.69 & -24.54 & & 251 & 0.15 & & & & \\
\hline $\mathrm{rms}$ & 20.1 & 0.92 & 0.99 & & 50 & 0.28 & & & & \\
\hline Inactive Es & $D(\mathrm{Mpc})$ & $M_{\mathrm{B}}(\mathrm{mag})$ & $M_{K_{\mathrm{S}}}(\mathrm{mag})$ & Class & $\sigma_{*}\left(\mathrm{~km} \mathrm{~s}^{-1}\right)$ & $A_{\mathrm{V}}(\mathrm{mag})$ & Profile & Ref. & Dust properties & Ref. \\
\hline NGC 221 & 0.7 & -15.51 & -19.13 & $\mathrm{~A}$ & 90 & 0.48 & PL & 3,12 & No dust & 3,12 \\
\hline NGC 821 & 23.2 & -20.11 & -23.93 & A & 205 & 0.31 & int & 1,4 & No dust & 3,4 \\
\hline NGC 2634 & 30.2 & -19.69 & -23.14 & A & 193 & 0.18 & PL & 1,8 & No dust & 6 \\
\hline NGC 3348 & 37.8 & -21.05 & -24.92 & A & 221 & 0.32 & core & 1,8 & No dust & 6 \\
\hline NGC 3377 & 8.1 & -18.47 & -22.10 & A & 166 & 0.36 & PL & 1,4 & Dust filaments & 4,6 \\
\hline NGC 3610 & 29.2 & -20.79 & -24.42 & A & 172 & 0.06 & PL & 1,4 & No dust & 4 \\
\hline NGC 3613 & 32.9 & -20.93 & -24.58 & A & 219 & 0.19 & core & 1,8 & No dust & 4 \\
\hline NGC 3640 & 24.2 & -20.73 & -24.40 & A & 196 & 0.27 & core & 1,4 & No dust & 4,6 \\
\hline NGC 4291 & 29.4 & -20.09 & -23.92 & A & 300 & 0.04 & core & 1,4 & No dust & $3,4,6$ \\
\hline NGC 4339 & 25.5 & -19.72 & -23.50 & A & 138 & -0.01 & & & & \\
\hline NGC 4365 & 16.8 & -20.64 & -24.49 & A & 251 & 0.30 & core & 1,4 & No dust & 4,6 \\
\hline NGC 4406 & 16.8 & -21.39 & -25.02 & A & 233 & 0.22 & core & 1,4 & No dust & 4 \\
\hline NGC 4473 & 16.8 & -20.10 & -23.97 & A & 176 & 0.18 & core & 1,4 & No dust & 4 \\
\hline NGC 4478 & 16.8 & -18.92 & -22.77 & A & 142 & 0.21 & core & 1,4 & No dust & 4,6 \\
\hline NGC 4564 & 16.8 & -19.17 & -23.19 & A & 160 & 0.24 & PL & 1,8 & No dust & 6,8 \\
\hline NGC 4621 & 16.8 & -20.60 & -24.38 & A & 245 & 0.14 & PL & 1,4 & No dust & 4,6 \\
\hline NGC 4648 & 27.5 & -19.49 & -23.35 & A & 222 & 0.26 & PL & 1,8 & Dust disk & 6,8 \\
\hline NGC 4649 & 16.8 & -21.43 & -25.39 & A & 349 & 0.23 & core & 1,4 & No dust & 4 \\
\hline NGC 4660 & 16.8 & -19.06 & -22.92 & A & 198 & 0.27 & PL & 1,4 & No dust & 4 \\
\hline NGC 4914 & 62.4 & -21.64 & -25.33 & A & 244 & -0.04 & & & & \\
\hline NGC 5557 & 42.6 & -21.17 & -25.07 & A & 290 & -0.20 & core & 1,4 & No dust & 4,6 \\
\hline NGC 5576 & 26.4 & -20.34 & -24.28 & A & 211 & 0.08 & core & 1,4 & No dust & 4,6 \\
\hline NGC 5638 & 28.4 & -20.21 & -24.01 & A & 152 & -0.32 & & & & \\
\hline NGC 5831 & 28.5 & -19.96 & -23.84 & A & 167 & 0.13 & PL & 1,8 & No dust & 6,8 \\
\hline NGC 7619 & 50.7 & -21.60 & -25.49 & A & 337 & 0.24 & core & 1,4 & No dust & 4 \\
\hline average & 25.7 & -20.11 & -23.90 & & 211 & 0.17 & & & & \\
\hline rms & 13.1 & 1.29 & 1.32 & & 62 & 0.17 & & & & \\
\hline
\end{tabular}

Column (5): nuclear spectral classification from the Palomar survey Ho et al. (1997a): $\mathrm{L}=\mathrm{LINER}, \mathrm{S}=$ Seyfert, $\mathrm{T}=$ transition object, and $\mathrm{A}=$ absorption-line nucleus (inactive). The last two rows show the average value and rms of each column.

References: 1. Lauer et al. (2007); 2. González Delgado et al. (2008); 3. Ravindranath et al. (2001); 4. Lauer et al. (2005); 5. Martel et al. (2004); 6. Tran et al. (2001); 7. van Dokkum \& Franx (1995); 8. Rest et al. (2001); 9. Quillen et al. (2000); 10. Laine et al. (2003); 11. Lauer et al. (1995); 12. Faber et al. (1997).

the full sample yields $P_{\text {null }} \approx 3 \%$, but the significance drops to $P_{\text {null }} \approx 9 \%$ after excluding NGC 315 and NGC 2832 from the LINER sample and NGC 221 from the inactive sample.

\subsection{Stellar populations}

As mentioned in Sect. 2, our spectral library covers 15 ages and 3 metallicities, which produce a total of 45 SSPs. Here we rebin the 45 SSPs into 5 components according to age: I $\left(10^{6} \leq t<10^{7} \mathrm{yr}\right)$; II $\left(10^{7} \leq t<10^{8} \mathrm{yr}\right)$; III $\left(10^{8} \leq t<10^{9} \mathrm{yr}\right)$; IV $\left(10^{9} \leq t<10^{10} \mathrm{yr}\right)$; and $\mathrm{V}\left(t \geq 10^{10} \mathrm{yr}\right)$. We derive the lightweighted mean stellar age using the formula in Cid Fernandes et al. (2005):

$\left\langle\log t_{*}\right\rangle_{\mathrm{L}}=\sum_{i=1}^{45} x_{i} \log t_{i}$, 


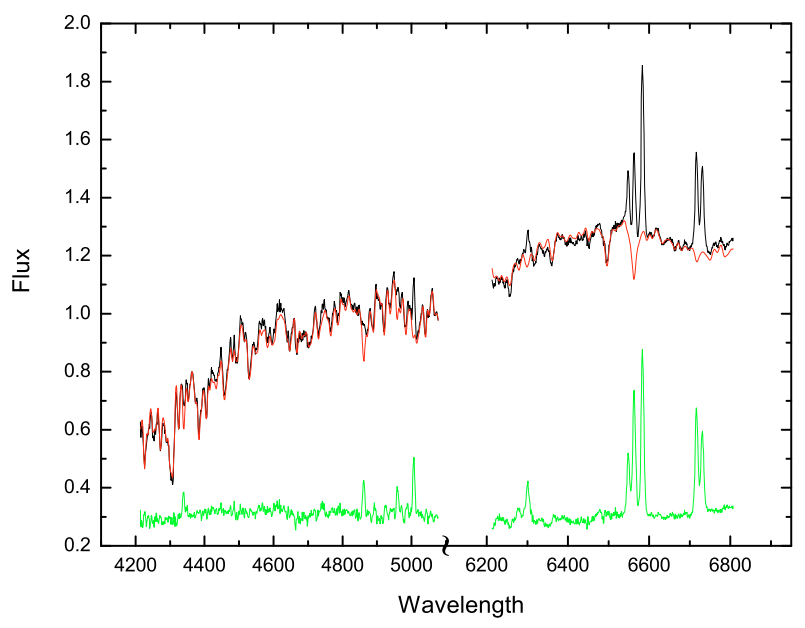

Fig. 1. Synthesis results for the LINER NGC 2768. The flux is normalized at $4750 \AA$. The black line is the observed spectrum; the red line is the synthesized spectrum; and the green line is the residual spectrum after subtracting the synthesized spectrum from the observed spectrum. The vertical scale has been shifted arbitrarily. There are no data in the region $5110-6210 \AA$.

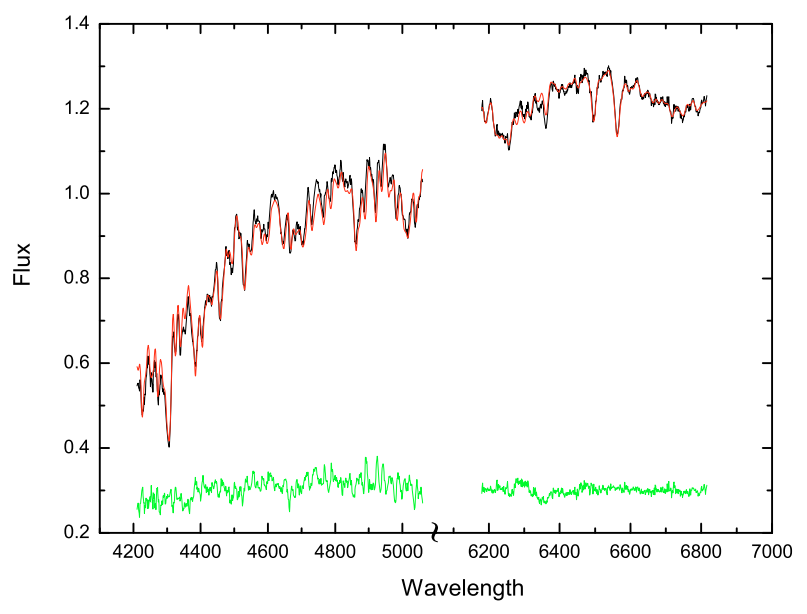

Fig. 2. Same as Fig. 1, but for the inactive elliptical NGC 4648.

where $x_{i}$ denotes the contribution of the $i$ th SSP and $t_{i}$ its corresponding age. Similarly, the light-weighted mean stellar metallicity is computed from

$$
\left\langle Z_{*}\right\rangle_{\mathrm{L}}=\sum_{i=1}^{45} x_{i} Z_{i},
$$

where $x_{i}$ represents the contribution of the $i$ th SSP with metallicity $Z_{i}$. The synthesis results are presented in Table 2 . Columns (2) through (6) give the fractional light contribution from the AGN PL component and from stellar populations I-V. Columns (7) and (8) list the mean stellar age and metallicity.

Figure 4 shows the contribution of each stellar population for the LINER (upper panel) and inactive E (lower panel) subsamples. It is clear that young and intermediate-age stellar populations contribute very little to the total flux for either group. For LINERs, stellar population I, II, and III contribute only $<1 \%$, $2 \%$, and $<1 \%$, respectively; for inactive Es, the corresponding values are $1 \%, 2 \%$, and $<1 \%$. Most of the optical light is dominated by an old population. The mean contribution from stellar population IV and V are $11 \%$ and $86 \%$ for LINERs, and $20 \%$ and $76 \%$ for inactive Es.
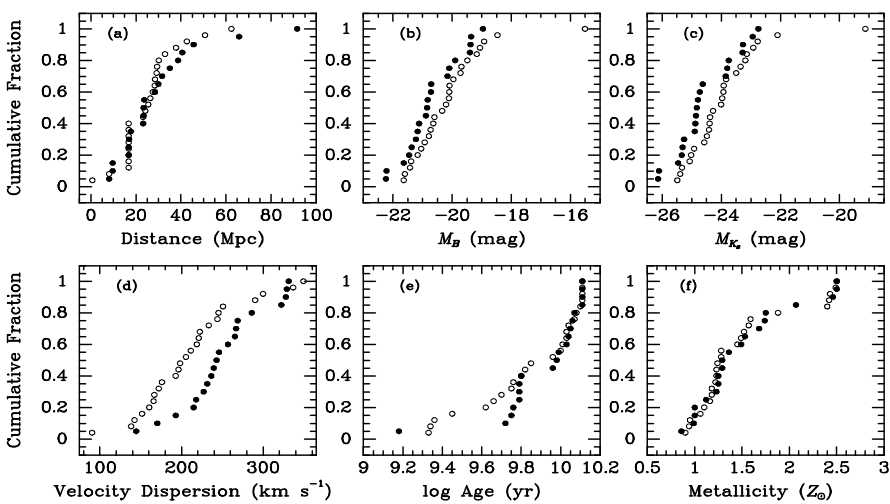

Fig. 3. Cumulative distribution of a) distance, b) absolute blue magnitude, c) absolute $K_{\mathrm{s}}$-band magnitude, d) central stellar velocity dispersion, e) mean stellar age, and f) mean stellar metallicity. Active (LINER) and inactive ellipticals are labeled as filled and open circles, respectively.

Figure $3 \mathrm{e}$ shows the cumulative distribution of mean stellar age for LINERs and inactive Es. Their average age is $8.1 \mathrm{Gyr}$ and $7.1 \mathrm{Gyr}$, respectively. The only exception is the LINER NGC 4374, which has a dominant 1.4 Gyr old stellar population that contributes $89 \%$ of the light. Our stellar population synthesis suggests that LINERs and inactive Es have very similar stellar populations, both lacking young and intermediate-age populations. A KS test yields $P_{\text {null }}=31 \%(68 \%$, NGC 221 excluded). The similarity of the stellar populations of the two groups further extends to the derived metallicities (Fig. 3f): the average metallicity for both subsamples is $\sim 1.5 Z_{\odot}$.

\subsection{Central light profile}

Central surface brightness profiles carry many clues on the formation and evolutionary history of the galaxy (Faber et al. 1997; Lauer et al. 2005). We compare the surface brightness profiles of LINERs and inactive Es derived from Hubble Space Telescope (HST) images, and try to find whether there is any difference between the two subsamples. The high angular resolution of HST is crucial to derive robust central profiles of galaxies.

Lauer et al. (1995) classified the central surface brightness profiles of early-type galaxies into two types. Core galaxies have central light distributions that turnover from a steep outer profile to a shallower cusp interior to some break radius. Power-law galaxies, on the other hand, display continuously rising inner profiles down to the resolution of $H S T$. The two classes correlate with the large-scale properties of the galaxies. Core galaxies are invariably luminous giant ellipticals that are slowly rotating and show boxy isophotes; power-law galaxies occupy more intermediate luminosities, rapidly rotate, and show disky isophotes. The physical implications of these two classes for their formation histories are discussed in detail in Faber et al. (1997).

Published HST surface brightness profiles and classifications are available for most of our sample, as documented in Table 1. Among the 16 LINERs with profile classifications, $14(88 \%)$ are core galaxies; only one galaxy, NGC 4494, is classified as a power-law system, and another, NGC 7626, has an intermediate profile. Four (NGC 315, 2768, 3226, and 5322) are too dusty to determine their profiles reliably and cannot be classified. However, for the inactive galaxies, only 12 out of $22(55 \%)$ have cores, whereas $9(41 \%)$ have power-law profiles; NGC 821 has an intermediate classification, and three others lack data. 
Table 2. Synthesis results.

\begin{tabular}{|c|c|c|c|c|c|c|c|c|}
\hline LINERs & $\overline{\text { PL (\%) }}$ & $\overline{\mathrm{I}(\%)}$ & II (\%) & III (\%) & IV (\%) & V(\%) & log age (yr) & Metallicity $\left(Z_{\odot}\right)$ \\
\hline NGC 315 & 7 & 2 & $<1$ & $<1$ & $<1$ & 92 & 9.79 & 1.29 \\
\hline NGC 1052 & $<1$ & $<1$ & $<1$ & $<1$ & 11 & 89 & 10.03 & 1.53 \\
\hline NGC 2768 & $<1$ & $<1$ & $<1$ & $<1$ & 37 & 63 & 9.79 & 2.07 \\
\hline NGC 2832 & $<1$ & $<1$ & $<1$ & $<1$ & $<1$ & 99 & 10.11 & 1.29 \\
\hline NGC 3193 & 9 & $<1$ & $<1$ & $<1$ & $<1$ & 91 & 9.72 & 1.12 \\
\hline NGC 3226 & $<1$ & $<1$ & $<1$ & $<1$ & 72 & 28 & 9.79 & 1.00 \\
\hline NGC 3379 & $<1$ & $<1$ & $<1$ & $<1$ & $<1$ & 99 & 10.11 & 1.68 \\
\hline NGC 3608 & 2 & $<1$ & $<1$ & $<1$ & $<1$ & 98 & 10.05 & 1.23 \\
\hline NGC 4261 & $<1$ & $<1$ & $<1$ & $<1$ & 5 & 95 & 10.06 & 1.49 \\
\hline NGC 4278 & $<1$ & 1 & $<1$ & $<1$ & $<1$ & 99 & 10.07 & 1.75 \\
\hline NGC 4374 & $<1$ & $<1$ & 2 & $<1$ & 90 & 8 & 9.18 & 2.46 \\
\hline NGC 4486 & $<1$ & 2 & 1 & $<1$ & $<1$ & 96 & 9.99 & 0.86 \\
\hline NGC 4494 & $<1$ & $<1$ & 7 & $<1$ & 11 & 83 & 9.80 & 1.25 \\
\hline NGC 4589 & $<1$ & $<1$ & 11 & $<1$ & 1 & 88 & 9.76 & 2.50 \\
\hline NGC 4636 & $<1$ & $<1$ & $<1$ & $<1$ & $<1$ & 99 & 10.04 & 1.00 \\
\hline NGC 5077 & $<1$ & 4 & $<1$ & $<1$ & $<1$ & 96 & 9.96 & 1.74 \\
\hline NGC 5322 & $<1$ & $<1$ & 12 & $<1$ & $<1$ & 88 & 9.75 & 2.50 \\
\hline NGC 5813 & $<1$ & $<1$ & $<1$ & $<1$ & $<1$ & 99 & 10.11 & 1.36 \\
\hline NGC 5982 & 1 & $<1$ & $<1$ & $<1$ & $<1$ & 98 & 9.98 & 0.99 \\
\hline NGC 7626 & $<1$ & $<1$ & $<1$ & $<1$ & $<1$ & 99 & 10.11 & 1.25 \\
\hline average & 1.0 & 0.4 & 1.7 & $<1$ & 11 & 86 & 9.91 & 1.52 \\
\hline $\mathrm{rms}$ & 2.5 & 1.0 & 3.6 & $<1$ & 25 & 24 & 0.22 & 0.51 \\
\hline Inactive Es & & $\mathrm{I}(\%)$ & II (\%) & III (\%) & IV (\%) & $\mathrm{V}(\%)$ & log age (yr) & Metallicity $\left(Z_{\odot}\right)$ \\
\hline NGC 221 & & 1 & $<1$ & 4 & 94 & $<1$ & 9.33 & 1.88 \\
\hline NGC 821 & & $<1$ & $<1$ & $<1$ & $<1$ & 99 & 10.10 & 1.18 \\
\hline NGC 2634 & & 1 & $<1$ & $<1$ & 1 & 98 & 10.07 & 1.28 \\
\hline NGC 3348 & & $<1$ & 4 & $<1$ & $<1$ & 96 & 10.01 & 2.50 \\
\hline NGC 3377 & & $<1$ & 8 & $<1$ & 11 & 82 & 9.34 & 2.49 \\
\hline NGC 3610 & & $<1$ & 7 & $<1$ & 56 & 37 & 9.36 & 2.40 \\
\hline NGC 3613 & & $<1$ & 3 & $<1$ & 24 & 73 & 9.80 & 1.57 \\
\hline NGC 3640 & & $<1$ & $<1$ & $<1$ & 30 & 70 & 9.82 & 1.45 \\
\hline NGC 4291 & & 4 & 2 & $<1$ & 29 & 65 & 9.70 & 2.42 \\
\hline NGC 4339 & & $<1$ & 3 & $<1$ & 1 & 95 & 9.96 & 1.59 \\
\hline NGC 4365 & & $<1$ & 12 & $<1$ & $<1$ & 88 & 9.75 & 1.28 \\
\hline NGC 4406 & & 2 & $<1$ & $<1$ & $<1$ & 98 & 10.04 & 0.94 \\
\hline NGC 4473 & & $<1$ & $<1$ & $<1$ & 29 & 71 & 9.85 & 1.49 \\
\hline NGC 4478 & & $<1$ & $<1$ & $<1$ & 99 & $<1$ & 9.66 & 0.90 \\
\hline NGC 4564 & & 3 & $<1$ & $<1$ & $<1$ & 97 & 10.00 & 1.10 \\
\hline NGC 4621 & & 2 & $<1$ & $<1$ & $<1$ & 98 & 10.03 & 1.18 \\
\hline NGC 4648 & & $<1$ & $<1$ & $<1$ & $<1$ & 99 & 10.11 & 1.23 \\
\hline NGC 4649 & & $<1$ & $<1$ & $<1$ & 3 & 96 & 10.03 & 0.95 \\
\hline NGC 4660 & & $<1$ & 5 & $<1$ & 15 & 80 & 9.76 & 1.23 \\
\hline NGC 4914 & & $<1$ & 8 & $<5$ & 87 & $<1$ & 9.45 & 2.43 \\
\hline NGC 5557 & & $<1$ & $<1$ & $<1$ & 1 & 99 & 10.08 & 1.06 \\
\hline NGC 5576 & & 2 & 6 & $<1$ & 25 & 66 & 9.62 & 1.52 \\
\hline NGC 5638 & & $<1$ & $<1$ & $<1$ & $<1$ & 99 & 10.11 & 1.16 \\
\hline NGC 5831 & & $<1$ & $<1$ & $<1$ & $<1$ & 99 & 10.11 & 1.22 \\
\hline NGC 7619 & & $<1$ & $<1$ & $<1$ & $<1$ & 99 & 10.11 & 1.24 \\
\hline average & & 0.6 & 2.3 & 0.4 & 20 & 76 & 9.85 & 1.51 \\
\hline $\mathrm{rms}$ & & 1.1 & 3.4 & 1.3 & 31 & 32 & 0.26 & 0.53 \\
\hline
\end{tabular}

Columns (2)-(7) list the fractional contribution to the total flux by the PL and by stellar populations I, II, III, IV, and V, respectively. Contributions less than $1 \%$ are listed as upper limits. Column (8) gives the mean stellar age, and Col. (9) gives the mean metallicity. The last two rows show the average value and rms of each column.

\subsection{Central dust content}

Elliptical galaxies are traditionally viewed as structurally simple systems composed of old stars and lacking in cold gas and dust. Recent observations, especially from HST images, show unambiguous evidence that many ellipticals exhibit central dust structures (van Dokkum \& Franx 1995; Tran et al. 2001; Lauer et al. 2005). Lauer et al. (2005) suggested a dust-settling sequence in early-type galaxies: the dust starts off poorly organized, then gradually settles down to the center and forms dusty rings or disks. The origin of gas and dust in elliptical galaxies is not well understood. Two possibilities are frequently discussed - an internal origin from stellar mass loss or an external origin from accretion of a gas-rich companion (Lauer et al. 2005; Sarzi et al. 2006). Although we do not have detailed kinematic information on our galaxies, they are morphologically regular and do not show obvious signs of recent interactions or mergers.

We compare the dust properties of LINERs and inactive Es in our sample. Information on the central dust properties, gathered from a literature search, is summarized in Table 1. Among 


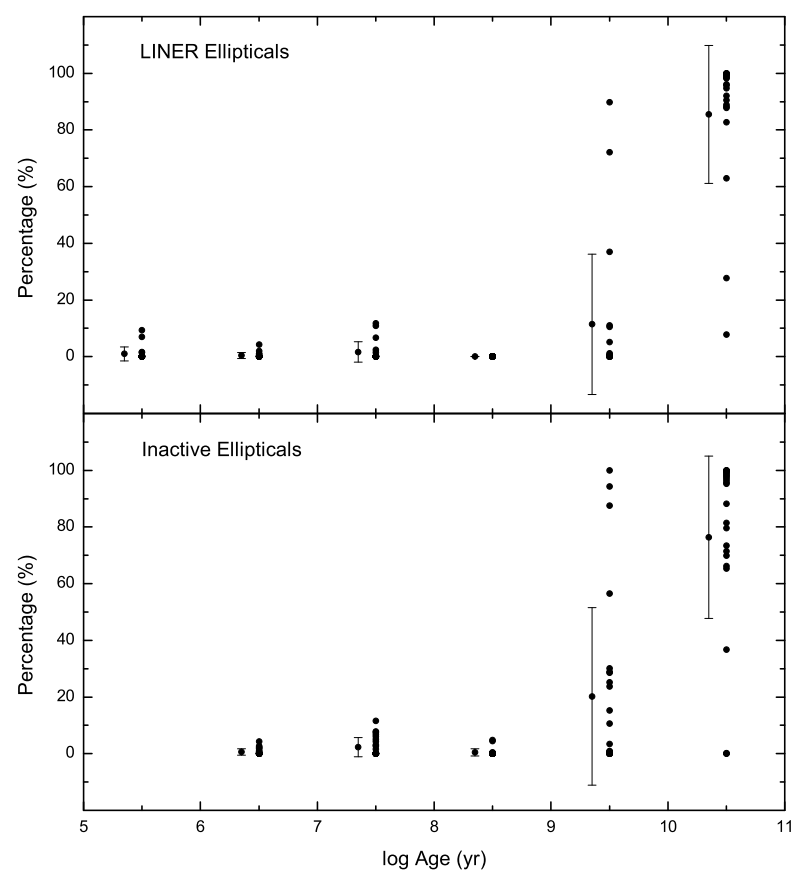

Fig. 4. The fractional contribution to the total flux of each stellar population for LINERs (upper panel) and inactive ellipticals (lower panel). The abscissa gives the logarithm of the age, where 5.5 corresponds to the AGN PL component; 6.5, 7.5, 8.5, 9.5, and 10.5 correspond to stellar populations I, II, III, IV, and V, respectively. The point and error bar to the left of each population indicate the average fraction and rms, respectively. We did not fit a PL component to the inactive ellipticals.

the LINERs, 16 out of 20 (80\%) have detected central dust structures. In stark contrast, only 2 out of $22(9 \%)$ inactive ellipticals with suitable $H S T$ data show dust features. Interestingly, the optical extinction derived from the population synthesis analysis $\left(A_{\mathrm{V}}\right.$ in Table 1$)$ shows no clear correspondence with the presence or absence of dust features inferred from the HST images; $\left\langle A_{\mathrm{V}}(\mathrm{LINERs})\right\rangle-\left\langle A_{\mathrm{V}}\right.$ (inactive $\left.)\right\rangle=-0.02 \pm 0.33$. This suggests that the dust features seen in the HST images lie on significantly smaller scales than the bulk of the stars sampled by the spectra.

\section{Discussion and conclusions}

Analyzing a statistically complete sample of 45 elliptical galaxies from the Palomar spectroscopic survey of nearby galaxies, comprising 20 LINERs and 25 inactive members, we show that galaxies hosting LINER nuclei, compared to systems lacking optical evidence for nuclear activity, have very similar stellar properties, both on global and nuclear scales. Specifically, we find that the two galaxy subgroups have essentially indistinguishable total optical and near-infrared luminosities, central stellar velocity dispersions, and luminosity-weighted mean stellar ages and metallicities on nuclear $(\$ 300 \mathrm{pc})$ scales. The lack of young or intermediate-age stars in LINERs strongly rule out starburst and post-starburst models for the excitation of LINER-like emission, further strengthening the proposition that LINERs are lowluminosity AGNs (Ho 2008).

Our results are qualitatively consistent with those from previous studies of the Palomar survey. Ho et al. (2003) systematically investigated the stellar population of the emission-line nuclei in the Palomar survey and found that LINERs generally contain evolved stellar populations. Other evidence concerning the old ages of the nuclear stellar population in nearby low-luminosity
AGNs is summarized in Ho (2008). Ho et al., however, did not derive detailed ages or metallicities from a population synthesis analysis. This work is the first quantitative analysis of its kind for the Palomar survey. On the other hand, studies of larger samples of LINERs drawn from the Sloan Digital Sky Survey (SDSS) reach somewhat different conclusions. While LINERs on average have older stars than Seyferts (Kewley et al. 2006), Graves et al. (2007) and Schawinski et al. (2007) show that LINERs have a tendency to exhibit somewhat younger populations (by $\sim 3$ Gyr) compared to inactive early-type galaxies devoid of emission lines. The differences between our results and those derived from SDSS can plausibly be attributed to a combination of several effects. First, the SDSS sources have redshifts $z \approx 0.05-0.1$, much more distant than the Palomar galaxies. As discussed by Ho (2008), aperture effects render the interpretation of LINER emission in distant galaxies somewhat problematic. Second, the stellar population analysis method of this paper differs from that used in the SDSS studies, and it is unclear if there are residual systematic discrepancies between the different methods. Third, our grid of SSP spectra may be too coarse to be able to resolve the small age difference reported in the SDSS studies. Finally, we note that the spectral range of the Palomar data is more limited than that of SDSS.

Surprisingly, we find that LINER host galaxies have a greater tendency to exhibit central core light profiles. Lauer et al. (2005) suggest that core galaxies are more luminous, more massive, and may be older than power-law galaxies, seemingly at odds with our results. Considering the modest size of our sample, however, and the large scatter in the statistical correlations between profile type and global properties, this disagreement is not too alarming.

By far the clearest distinction between active and inactive ellipticals is that the former has a much higher likelihood of containing circumnuclear dust. This finding is in excellent agreement with that of Lauer et al. (2005), who also found a strong correlation between the incidence of nebular line emission which in ellipticals invariably signifies a LINER classification (Ho 2008) - and central dust features: $90 \%$ of emission-line nuclei show detected central dust structures, to be compared with just 4\% for the lineless systems. Ravindranath et al. (2001) similarly suggested a connection between $\mathrm{H} \alpha$ emission and dust features, and the tendency for LINERs to be more dusty. Recently, Simões Lopes et al. (2007) presented strong evidence that circumnuclear dust is correlated with AGN activity in early-type galaxies. Among 34 early-type galaxies classified as AGNs, all show circumnuclear dust structures, to be compared with only $26 \%$ in the control sample of inactive systems.

Taken collectively, the above evidence suggests that ellipticals with weakly active, LINER nuclei are more gas-rich than their inactive counterparts. Given the lower stellar density in the cores, we actually expect less cold gas accumulation from stellar mass loss, not more, among the LINER hosts (see, e.g., Soria et al. 2006). On the other hand, massive ellipticals are surrounded by hot X-ray halos (e.g., Jones et al. 2002), whose cooling time is sufficiently short that cooling flows should develop. We speculate that the central dust features in core-type LINER ellipticals may partly originate from cooling condensations in the X-ray-emitting gas. The "extra" cold gas and dust, in combination with direct spherical accretion from the hot gas itself, may be responsible for sustaining the weak nuclear activity. Perhaps elliptical galaxies with LINER nuclei identify the subclass of early-type galaxies in which this mode of accretion is significant.

Acknowledgements. We thank an anonymous referee for comments that led to significant improvements in the paper. The starlight project is supported 
by the Brazilian agencies CNPq, CAPES and FAPESP and by the FranceBrazil CAPES/Cofecub program. This work is supported by Program for New Century Excellent Talents in University (NCET), the National Natural Science Foundation of China under grants 10221001 and 10633040, the National Basic Research Program (973 program No. 2007CB815405), and the Carnegie Institution of Washington. This research has made use of NASA's Astrophysics Data System Bibliographic Services and the NASA/IPAC Extragalactic Database (NED), which is operated by the Jet Propulsion Laboratory, California Institute of Technology, under contract with the National Aeronautics and Space Administration. This publication makes use of data products from the Two Micron All Sky Survey, which is a joint project of the University of Massachusetts and the Infrared Processing and Analysis Center/California Institute of Technology, funded by the National Aeronautics and Space Administration and the National Science Foundation.

\section{References}

Barth, A. J., Filippenko, A. V., \& Moran, E. C. 1999a, ApJ, 515, L61 Barth, A. J., Filippenko, A. V., \& Moran, E. C. 1999b, ApJ, 525, 673 Barth, A. J., Ho, L. C., Filippenko, A. V., Rix, H.-W., \& Sargent, W. L. W. 2001, ApJ, 546, 205

Bruzual, G., \& Charlot, S. 2003, MNRAS, 344, 1000

Cardelli, J. A., Clayton, G. C., \& Mathis, J. S. 1989, ApJ, 345, 245

Cid Fernandes, R., Gu, Q., Melnick, J., et al. 2004, MNRAS, 355, 273

Cid Fernandes, R., Mateus, A., Sodré, L., Stasińska, G., \& Gomes, J. M. 2005, MNRAS, 358, 363

Faber, S. M., Tremaine, S., Ajhar, E. A., et al. 1997, AJ, 114, 1771

Ferguson, H. C., \& Binggeli, B. 1994, A\&AR, 6, 67

Ferrarese, L., \& Merritt, D. 2000, ApJ, 539, L9

Gebhardt, K., Bender, R., Bower, G., et al. 2000, ApJ, 539, L13

González Delgado, R. M., Pérez, E., Cid Fernandes, R., \& Schmitt, H. 2008, AJ, 135,747

Graves, G. J., Faber, S. M., Schiavon, R. P., \& Yan, R. 2007, ApJ, 671, 243

Gu, Q., Melnick, J., Cid Fernandes, R., et al. 2006, MNRAS, 366, 480

Heckman, T. M. 1980, A\&A, 87, 152

Ho, L. C. 2008, ARA\&A, in press [arXiv:0803.2268]
Ho, L. C., Filippenko, A. V., \& Sargent, W. L. W. 1995, ApJS, 98, 477

Ho, L. C., Filippenko, A. V., \& Sargent, W. L. W. 1997a, ApJS, 112, 315

Ho, L. C., Filippenko, A. V., Sargent, W. L. W., \& Peng, C. Y. 1997b, ApJS, 112, 391

Ho, L. C., Rudnick, G., Rix, H.-W., et al. 2000, ApJ, 541, 120

Ho, L. C., Feigelson, E. D., Townsley, L. K., et al. 2001, ApJ, 549, L51

Ho, L. C., Filippenko, A. V., \& Sargent, W. L. W. 2003, ApJ, 583, 159

Jones, C., Forman, W., Vikhlinin, A., et al. 2002, ApJ, 567, L115

Kaneda, H., Onaka, T., \& Sakon, I. 2005, ApJ, 632, L83

Kewley, L. J., Groves, B., Kauffmann, G., \& Heckman, T. 2006, MNRAS, 372 , 961

Knapp, G. R., Guhathakurta, P., Kim, D.-W., \& Jura, M. A. 1989, ApJS, 70, 329 Kormendy, J. 1985, ApJ, 295, 73

Laine, S., van der Marel, R. P., Lauer, T. R., et al. 2003, AJ, 125, 478

Lauer, T. R., Ajhar, E. A., Byun, Y.-I., et al. 1995, AJ, 110, 2622

Lauer, T. R., Faber, S. M., Gebhardt, K., et al. 2005, AJ, 129, 2138

Lauer, T. R., Gebhardt, K., Faber, S. M., et al. 2007, ApJ, 664, 226

Maoz, D., Nagar, N. M., Falcke, H., \& Wilson, A. S. 2005, ApJ, 625, 699

Martel, A. R., Ford, H. C., Bradley, L. D., et al. 2004, AJ, 128, 2758

Nagar, N. M., Falcke, H., \& Wilson, A. S. 2005, A\&A, 435, 521

Phillips, M. M., Jenkins, C. R., Dopita, M. A., Sadler, E. M., \& Binette, L. 1986, AJ, 91, 1062

Quillen, A. C., Bower, G. A., \& Stritzinger, M. 2000, ApJS, 128, 85

Ravindranath, S., Ho, L. C., Peng, C. Y., Filippenko, A. V., \& Sargent, W. L. W. 2001, AJ, 122, 653

Rest, A., van den Bosch, F. C., Jaffe, W., et al. 2001, AJ, 121, 2431

Sarzi, M., Falcón-Barroso, J., Davies, R. L., et al. 2006, MNRAS, 366, 1151

Schawinski, K., Thomas, D., Sarzi, M., et al. 2007, MNRAS, 382, 1415

Soria, R., Graham, A. W., Fabbiano, G., et al. 2006, ApJ, 640, 143

Shields, J. C. 1991, AJ, 102, 1314

Shields, J. C., Rix, H.-W., McIntosh, D. H., et al. 2000, ApJ, 534, L27

Simões Lopes, R. D., Storchi-Bergmann, T., de Fátima de, O., Saraiva, M., \& Martini, P. 2007, ApJ, 655, 718

Skrutskie, M. F., Cutri, R. M., Stiening, R., et al. 2006, AJ, 131, 1163

Temi, P., Brighenti, F., Mathews, W. G., \& Bregman, J. D. 2004, ApJS, 151, 237

Terashima, Y., \& Wilson, A. S. 2003, ApJ, 583, 145

Tran, H. D., Tsvetanov, Z., Ford, H. C., et al. 2001, AJ, 121, 2928

van Dokkum, P. G., \& Franx, M. 1995, AJ, 110, 2027 